\title{
Semiglobal Finite-Time Synchronization of Complex Dynamical Networks via Periodically Intermittent Control
}

\author{
Yihan Fan, Hongmei Liu, and Jun Mei \\ College of Science, China Three Gorges University, Yichang, Hubei 443002, China \\ Correspondence should be addressed to Yihan Fan; fanyihan071703@163.com
}

Received 7 October 2014; Accepted 21 April 2015

Academic Editor: Agacik Zafer

Copyright (C) 2015 Yihan Fan et al. This is an open access article distributed under the Creative Commons Attribution License, which permits unrestricted use, distribution, and reproduction in any medium, provided the original work is properly cited.

\begin{abstract}
This paper studies the finite-time synchronization problem for a class of complex dynamical networks by means of periodically intermittent control. Based on some analysis techniques and finite-time stability theory, some novel and effective finite-time synchronization criteria are given in terms of a set of linear matrix inequalities. Particularly, the previous synchronization problem by using periodically intermittent control has been extended in this paper. Finally, numerical simulations are presented to verify the theoretical results.
\end{abstract}

\section{Introduction}

A complex dynamical network consists of a number of nodes, which are dynamic systems, and links between the nodes. Complex networks exist in various fields of science, engineering, and society and have been deeply investigated in recent years [1]. As the major collective behavior, synchronization is one of the key issues that has been extensively addressed. Several books and reviews [2-6] have appeared which deal with this topic.

Up till now, the synchronization for nonlinear systems especially dynamical networks $[7,8]$ has been one of the extensive research subjects and many important and fundamental results have been reported on the synchronization and control of nonlinear systems. Meanwhile, lots of control approaches have been developed to synchronize nonlinear systems such as adaptive control $[9,10]$, feedback control $[11,12]$, observer control $[13,14]$, impulsive control [1519], and intermittent control [20-26]. Among these control approaches and other control methods, the discontinuous control methods, such as impulsive control and intermittent control, have received much interest because they are practical and easily implemented in engineering fields such as transportation and communication. Though the two control methods are discontinuous control, the intermittent control is different from the impulsive control since impulsive control is activated only at some isolated instants, while intermittent control has a nonzero control width. Under some circumstances, using intermittent control is more effective and robust [27]. Hence, some synchronization criteria for nonlinear systems with or without time delays via intermittent control have been presented in recent years; see [21, 22 , $28,29]$.

Nevertheless, to our best knowledge, the previous results only focus on asymptotical or exponential synchronization of networks through intermittent control; there are few results concerned with finite-time synchronization via intermittent control. In view of this, the purpose of this paper is to study the synchronization of a class of systems by designing reasonable intermittent control. In addition, some previous work views the finite-time synchronization via intermittent control in [30], which will be extended in this paper. Besides, many superiority in finite-time stability has no emphasis in this paper (see [30-32]).

The main contribution of this paper lies in the following aspects. Firstly, a new central lemma is proved by using analysis method. Additionally, an intermittent controller is designed to synchronize the addressed complex networks and some new and useful finite-time synchronization criteria are 
obtained. Besides, the derivative of the Lyapunov function $V(t)$ is smaller than $-\alpha V^{\eta}(t)+\beta V(t)$, which enriches the previous results in [30], when controllers are added into the network. Finally, numerical examples are given to show the effectiveness of the theoretical results.

The paper is organized as follows. In Section 2, the problem statement and synchronization scheme to be studied are formulated, and some useful lemmas and preliminaries are presented. In Section 3, some finite-time synchronization criteria for the complex dynamical networks are rigorously derived. In Section 4, the effectiveness of the developed methods is shown by numerical examples. Conclusions are finally drawn in Section 5.

\section{Preliminaries}

Consider a complex dynamical network consisting of $N$ linearly and diffusively coupled identical nodes, with each node being an $n$-dimensional dynamical system. The state equation of the entire network is designed as follows:

$$
\dot{x}_{i}(t)=f\left(x_{i}(t)\right)+c \sum_{j=1}^{N} a_{i j} \Gamma x_{j}(t), \quad i=1,2, \ldots, N
$$

where $x_{i}(t)=\left(x_{i 1}(t), x_{i 2}(t), \ldots, x_{i n}(t)\right)^{T} \in R^{n}$ is the state vector of the $i$ th dynamical node, $f: R^{n} \rightarrow R^{n}$ is a smooth nonlinear vector-value function, and the constant $c>0$ is a coupling strength. $\Gamma \in R^{n \times n}$ is the inner-coupling matrix of the network. Matrix $A=\left(a_{i j}\right) \in R^{N \times N}$ represents the coupling configuration of the network, in which $a_{i j}$ is defined as follows: if there is a connection from the nodes $j$ to $i$, then $a_{i j} \neq 0$; otherwise, $a_{i j}=0$, and the diagonal elements of matrices $A$ are defined as

$$
a_{i i}=-\sum_{j=1, j \neq i}^{N} a_{i j}
$$

To achieve the aim of this paper, the following assumptions and some lemmas are necessary.

Assumption 1. Assume that there exists a positive definite diagonal matrix $P=\operatorname{diag}\left(p_{1}, \ldots, p_{n}\right)$ and a diagonal matrix $\Theta=\operatorname{diag}\left(\theta_{1}, \ldots, \theta_{n}\right)$, such that $f(\cdot)$ satisfies the following inequality:

$$
\begin{aligned}
& (y-x)^{T} P(f(y)-f(x)-\Theta(y-x)) \\
& \leq-\xi(y-x)^{T}(y-x),
\end{aligned}
$$

for some $\xi>0$, all $x, y \in R^{n}$, and $t>0$.
Lemma 2 (see [33]). Assume that a continuous, positivedefinite function $V(t)$ satisfies the following differential inequality:

$$
\dot{V}(t) \leq-\alpha V^{\eta}(t), \quad \forall t \geq t_{0}, V\left(t_{0}\right) \geq 0
$$

where $\alpha>0$ and $0<\eta<1$ are all constants. Then, for any given $t_{0}, V(t)$ satisfies the following inequality:

$$
\begin{aligned}
V^{1-\eta}(t) & \leq V^{1-\eta}\left(t_{0}\right)-\alpha(1-\eta)\left(t-t_{0}\right), \quad t_{0} \leq t \leq t_{1}, \\
V(t) & \equiv 0, \quad \forall t \geq t_{1},
\end{aligned}
$$

with $t_{1}$ given by

$$
t_{1}=t_{0}+\frac{V^{1-\eta}\left(t_{0}\right)}{\alpha(1-\eta)}
$$

Lemma 3. Assume that a continuous, positive-definite function $V(t)$ satisfies the following differential inequality:

$$
\dot{V}(t) \leq-\alpha V^{\eta}(t)+p V(t), \quad \forall t \geq t_{0}, V^{1-\eta}\left(t_{0}\right) \leq \frac{\alpha}{p}
$$

where $\alpha>0,0<\eta<1$, and $p>0$ are three constants. Then, for any given $t_{0}, V(t)$ satisfies the following inequality:

$$
\begin{aligned}
& V^{1-\eta}(t) \exp \{-(1-\eta) p t\} \\
& \leq V^{1-\eta}\left(t_{0}\right) \exp \left\{-(1-\eta) p t_{0}\right\} \\
& +\frac{\alpha}{p}\left[\exp \{-(1-\eta) p t\}-\exp \left\{-(1-\eta) p t_{0}\right\}\right], \\
& \quad t_{0} \leq t \leq t_{1}, \\
& \text { with } t_{1} \text { given by } \quad \forall t \geq t_{1}, \\
& t_{1}=\frac{\ln \left(1-(p / \alpha) V^{1-\eta}(0)\right)}{p(\eta-1)}
\end{aligned}
$$

for $t_{0}=0$.

Proof. Consider the following differential equation:

$$
\begin{aligned}
\dot{X}(t) & =-\alpha X^{\eta}(t)+p X(t), \\
X\left(t_{0}\right) & =V\left(t_{0}\right)
\end{aligned}
$$

By multiplying $\exp \{-p t\}$, we have

$$
\begin{aligned}
& \frac{d(\exp \{-p t\} X(t))}{d t} \\
& =-\alpha(\exp \{-p t\} X(t))^{\eta} \exp \{-(1-\eta) p t\} .
\end{aligned}
$$


Although this differential equation does not satisfy the global Lipschitz condition, the unique solution to this equation can be found as

$$
\begin{aligned}
X^{1-\eta}(t) \exp \{-(1-\eta) p t\} \\
=X^{1-\eta}\left(t_{0}\right) \exp \left\{-(1-\eta) p t_{0}\right\} \\
\quad+\frac{\alpha}{p}\left[\exp \{-(1-\eta) p t\}-\exp \left\{-(1-\eta) p t_{0}\right\}\right], \\
X(t) \equiv 0, \quad \forall t \geq t_{1} .
\end{aligned}
$$

It is direct to prove that $x(t)$ is differential for $t>t_{0}$. From the comparison lemma, one obtains

$$
\begin{aligned}
& V^{1-\eta}(t) \exp \{-(1-\eta) p t\} \\
& \leq V^{1-\eta}\left(t_{0}\right) \exp \left\{-(1-\eta) p t_{0}\right\} \\
& \quad+\frac{\alpha}{p}\left[\exp \{-(1-\eta) p t\}-\exp \left\{-(1-\eta) p t_{0}\right\}\right], \\
& \quad t_{0} \leq t \leq t_{1}, \\
& V(t) \equiv 0, \quad \forall t \geq t_{1}
\end{aligned}
$$

with $t_{1}$ given in (9) with $t_{0}=0$.

Remark 4. Lemma 3 is similar to Lemma 2, but our result can enrich the famous differential inequality [33] to general differential inequality, and give a direction to proof the following Lemma 5.

Lemma 5. Suppose that function $V(t)$ is continuous and nonnegative when $t \in[0, \infty)$ and satisfies the following conditions:

$$
\begin{aligned}
& \dot{V}(t) \leq-\alpha V^{\eta}(t)+p V(t), \quad l T \leq t<l T+\theta T, \\
& \dot{V}(t) \leq 0, \quad l T+\theta T \leq t<(l+1) T,
\end{aligned}
$$

where $\alpha>0, T>0, p>0,0<\eta, \theta<1, l \in \iota=\{1,2, \ldots, \varsigma\}$, and $\varsigma$ is a nature number; then the following inequality holds:

$$
\begin{aligned}
& V^{1-\eta}(t) \exp \{-(1-\eta) p t\} \\
& \quad \leq V^{1-\eta}(0)+\frac{\alpha}{p}[\exp \{-(1-\eta) p \theta t\}-1],
\end{aligned}
$$

$$
0 \leq t \leq \widetilde{T}
$$

Proof. Denote $M_{0}=V^{1-\eta}(0)-\alpha / p$ and $W(t)=$ $V^{1-\eta}(t) \exp \{-(1-\eta) p t\}$, where $t \geq 0$. Let $Q(t)=W(t)-M_{0}-$ $(\alpha / p) \exp \{-(1-\eta) p t\}$. It is easy to see that

$$
Q(t)<0, \quad \text { for } t=0 \text {. }
$$

In the following, we will prove that

$$
Q(t)<0, \quad \forall t \in[0, \theta T)
$$

Otherwise, there exists a $t_{0} \in[0, \theta T)$ such that

$$
\begin{aligned}
& Q\left(t_{0}\right)=0, \\
& \dot{Q}\left(t_{0}\right)>0, \\
& Q(t)<0, \quad 0 \leq t<t_{0} .
\end{aligned}
$$

From (16), (18), and (19), we obtain

$$
\begin{aligned}
& \dot{Q}\left(t_{0}\right)=(1-\eta) V^{-\eta}\left(t_{0}\right) \dot{V}\left(t_{0}\right) \exp \left\{-(1-\eta) p t_{0}\right\} \\
& +V^{1-\eta}\left(t_{0}\right)\left[-(1-\eta) p \exp \left\{-(1-\eta) p t_{0}\right\}\right] \\
& -\left[\frac{\alpha}{p} \exp \left\{-(1-\eta) p t_{0}\right\}(-(1-\eta) p)\right] \leq(1-\eta) \\
& \cdot V^{-\eta}\left(t_{0}\right)\left(-\alpha V^{\eta}\left(t_{0}\right)+p V\left(t_{0}\right)\right) \\
& \cdot \exp \left\{-(1-\eta) p t_{0}\right\}-p(1-\eta) V^{1-\eta}\left(t_{0}\right) \\
& \cdot \exp \left\{-(1-\eta) p t_{0}\right\}+\alpha(1-\eta) \\
& \cdot \exp \left\{-(1-\eta) p t_{0}\right\}=-\alpha(1-\eta) \\
& \quad \cdot \exp \left\{-(1-\eta) p t_{0}\right\}+\alpha(1-\eta) \\
& \cdot \exp \left\{-(1-\eta) p t_{0}\right\}+p(1-\eta) V^{1-\eta}\left(t_{0}\right) \\
& \cdot \exp \left\{-(1-\eta) p t_{0}\right\}-p(1-\eta) V^{1-\eta}\left(t_{0}\right) \\
& \cdot \exp \left\{-(1-\eta) p t_{0}\right\}=0,
\end{aligned}
$$

which leads to a contradiction with (18). Hence inequality (17) holds.

Now, we prove that for $t \in[\theta T, T)$

$$
\begin{aligned}
& H(t) \\
& =W(t)-M_{0} \\
& -\frac{\alpha}{p} \exp \{-(1-\eta) p t\} \exp \{(1-\eta) p(t-\theta T)\} \\
& <0 \text {. }
\end{aligned}
$$

Otherwise, there exists a $t_{1} \in[\theta T, T)$ such that

$$
\begin{aligned}
H\left(t_{1}\right) & =0, \\
\dot{H}\left(t_{1}\right) & >0, \\
H(t) & <0, \quad \theta T \leq t<t_{1} .
\end{aligned}
$$


By (22) and (23), we have

$$
\begin{aligned}
& \dot{H}\left(t_{1}\right)=\dot{W}\left(t_{1}\right)-\frac{\alpha}{p} \exp \left\{-(1-\eta) p t_{1}\right\}(-(1-\eta) p) \\
& \cdot \exp \left\{(1-\eta) p\left(t_{1}-\theta T\right)\right\}-\frac{\alpha}{p} \exp \left\{-(1-\eta) p t_{1}\right\} \\
& \cdot \exp \left\{(1-\eta) p\left(t_{1}-\theta T\right)\right\}((1-\eta) p) \leq \alpha(1-\eta) \\
& \cdot \exp \left\{-(1-\eta) p t_{1}\right\} \exp \left\{(1-\eta) p\left(t_{1}-\theta T\right)\right\} \\
& -\alpha(1-\eta) \exp \left\{-(1-\eta) p t_{1}\right\} \\
& \cdot \exp \left\{(1-\eta) p\left(t_{1}-\theta T\right)\right\}=0,
\end{aligned}
$$

which contradicts (22). Hence (21) holds.

Consequently, on the one hand, for $t \in[\theta T, T)$,

$$
\begin{aligned}
& W(t) \\
& <M_{0} \\
& \quad+\frac{\alpha}{p} \exp \{-(1-\eta) p t\} \exp \{(1-\eta) p(t-\theta T)\} \\
& \leq M_{0} \\
& \quad+\frac{\alpha}{p} \exp \{-(1-\eta) p t\} \exp \{(1-\eta) p(1-\theta) T\} .
\end{aligned}
$$

On the other hand, it follows from (16) and (17) that for $t \in$ $[0, \theta T)$

$$
\begin{aligned}
& W(t) \\
& <M_{0}+\frac{\alpha}{p} \exp \{-(1-\eta) p t\} \\
& \leq M_{0} \\
& \quad+\frac{\alpha}{p} \exp \{-(1-\eta) p t\} \exp \{(1-\eta) p(1-\theta) T\} .
\end{aligned}
$$

So

$W(t)$

$$
\begin{array}{r}
<M_{0} \\
+\frac{\alpha}{p} \exp \{-(1-\eta) p t\} \exp \{(1-\eta) p(1-\theta) T\}, \\
\forall t \in[0, T) .
\end{array}
$$

Similarly, we can prove the following results for $t \in[T,(1+$ $\theta) T)$

$$
\begin{aligned}
& W(t) \\
& <M_{0} \\
& \quad+\frac{\alpha}{p} \exp \{-(1-\eta) p t\} \exp \{(1-\eta) p(1-\theta) T\},
\end{aligned}
$$

and for $t \in[(1+\theta) T, 2 T)$

$$
\begin{aligned}
& W(t)<M_{0}+\frac{\alpha}{p} \exp \{-(1-\eta) p t\} \\
& \cdot \exp \{(1-\eta) p(1-\theta) T\} \\
& \cdot \exp \{(1-\eta) p(t-\theta T-T)\}=M_{0}+\frac{\alpha}{p} \\
& \cdot \exp \{-(1-\eta) p t\} \exp \{(1-\eta) p(t-2 \theta T)\} .
\end{aligned}
$$

Now, using mathematical induction method, suppose that the following statements are true; for any integers $m$, we can obtain $W(t)$.

For $m T \leq t<(m+\theta) T$,

$$
\begin{aligned}
W(t)<M_{0}+\frac{\alpha}{p} \exp \{-(1-\eta) p t\} \\
\cdot \exp \{(1-\eta) p(1-\theta) m T\},
\end{aligned}
$$

and for $(m+\theta) T \leq t<(m+1) T$,

$$
\begin{aligned}
W(t)<M_{0}+\frac{\alpha}{p} \exp \{-(1-\eta) p t\} \\
\cdot \exp \{(1-\eta) p(t-(m+1) \theta T)\} .
\end{aligned}
$$

Since, for any $t \geq 0$, there exists a positive integer $k$, such that $k T \leq t<(k+1) T$, we can conclude the following estimation of $W(t)$ by (30) and (31).

For $k T \leq t<(k+\theta) T$,

$W(t)$

$$
<M_{0}
$$$$
+\frac{\alpha}{p} \exp \{-(1-\eta) p t\} \exp \{(1-\eta) p(1-\theta) k T\}
$$

$$
\begin{aligned}
\leq & M_{0} \\
& +\frac{\alpha}{p} \exp \{-(1-\eta) p t\} \exp \{(1-\eta) p(1-\theta) t\},
\end{aligned}
$$

and for $(k+\theta) T \leq t<(k+1) T$,

$$
\begin{aligned}
& W(t)<M_{0}+\frac{\alpha}{p} \exp \{-(1-\eta) p t\} \\
& \cdot \exp \{(1-\eta) p(t-(k+1) \theta T)\} \leq M_{0}+\frac{\alpha}{p} \\
& \cdot \exp \{-(1-\eta) p t\} \exp \{(1-\eta) p(1-\theta) t\} .
\end{aligned}
$$


From the previous definition of $W(t)$, we have

$$
\begin{aligned}
& V^{1-\eta}(t) \exp \{-(1-\eta) p t\} \\
& \leq V^{1-\eta}(0)-\frac{\alpha}{p} \\
& \quad+\frac{\alpha}{p} \exp \{-(1-\eta) p t\} \exp \{(1-\eta) p(1-\theta) t\} \\
& =V^{1-\eta}(0)-\frac{\alpha}{p}+\frac{\alpha}{p} \exp \{-(1-\eta) \theta p t\} \\
& =V^{1-\eta}(0)+\frac{\alpha}{p}[\exp \{-(1-\eta) p \theta t\}-1], \quad t \geq 0 .
\end{aligned}
$$

The proof of Lemma 3 is completed.

Remark 6. Lemmas 3 and 5 played an important role in the finite-time synchronization analysis of dynamical networks via intermittent control in this paper, because it shows the utilization of finite-time intermittent control.

Lemma 7 (see [30]). Let $x_{1}, x_{2}, \ldots, x_{n} \in R^{m}$ be any vectors and $0<q<2$ is a real number satisfying

$$
\begin{aligned}
& \left\|x_{1}\right\|^{q}+\left\|x_{2}\right\|^{q}+\cdots+\left\|x_{n}\right\|^{q} \\
& \quad \geq\left(\left\|x_{1}\right\|^{2}+\left\|x_{2}\right\|^{2}+\cdots+\left\|x_{n}\right\|^{2}\right)^{q / 2} .
\end{aligned}
$$

\section{Criteria for Finite-Time Synchronization}

In this section, we study finite-time synchronization of system (1) with system (36) under the following intermittent controller (37).

In order to drive system (1) to achieve finite-time synchronization by means of periodically intermittent control, the corresponding response system is designed as follows:

$$
\begin{aligned}
\dot{y}_{i}(t)=f\left(y_{i}(t)\right)+c \sum_{j=1}^{N} a_{i j} \Gamma y_{j}(t)+u_{i}(t) & \\
& \\
& i=1,2, \ldots, N,
\end{aligned}
$$

where $y_{i}(t)=\left(y_{i 1}(t), y_{i 2}, \ldots, y_{i n}(t)\right)^{T} \in R^{n}, i=1,2, \ldots, N$, denote the response state vector of the node $i$ of system (36). $u(t)=\left(u_{1}(t), u_{2}(t), \ldots, u_{N}(t)\right)^{T}$ is an intermittent controller defined as follows:

$$
\begin{aligned}
& u_{i}(t)=-\eta_{i} e_{i}(t)-\bar{k} \frac{\sqrt{\lambda_{\max }(P)}}{\lambda_{\min }(P)} \operatorname{sign}\left(e_{i}\right) \text {, } \\
& l T \leq t<l T+\delta, \\
& u_{i}(t)=0, \quad\left\|e_{i}\right\|=0 \text { or } l T+\delta \leq t<(l+1) T,
\end{aligned}
$$

where $\eta_{i}>0$ is a positive constant control gain and $\bar{k}>0$ is a tunable constant. Denote $\lambda_{\max }(P)\left(\lambda_{\min }(P)\right)$ as the maximum (minimum) eigenvalue of the matrix $P . T>0$ is the control period, $\delta>0$ is called the control width (control duration), and $\theta=\delta / T$ is the ratio of the control width $\delta$ to the control period $T$ called control rate. $\iota=\{1,2, \ldots, \varsigma\}$ is a finite natural number set and $l \in l$.

Let $e_{i}(t)=y_{i}(t)-x_{i}(t)(1 \leq i \leq N)$ be synchronization errors between the states of drive system (1) and response system (37); then the following error system can be obtained:

$$
\begin{gathered}
\dot{e}_{i}(t)=f\left(y_{i}(t)\right)-f\left(x_{i}(t)\right)+c \sum_{j=1}^{N} a_{i j} \Gamma e_{j}(t)+u_{i}(t), \\
l T \leq t<l T+\theta T, i=1,2, \ldots, N, \\
\dot{e}_{i}(t)=f\left(y_{i}(t)\right)-f\left(x_{i}(t)\right)+c \sum_{j=1}^{N} a_{i j} \Gamma e_{j}(t), \\
l T+\theta T \leq t<(l+1) T, i=1,2, \ldots, N .
\end{gathered}
$$

The main results are stated as follows.

Theorem 8. Let Assumption 1 hold. Suppose that positive constants $\eta_{1}, \eta_{2}, \ldots, \eta_{N}, \xi, \beta$, and a positive defined diagonal matrix $P>0$ satisfy

$$
\begin{array}{r}
\theta_{j} I_{N}-\Xi+c \gamma_{j} A-\beta I_{N} \leq 0, \quad j=1,2, \ldots, n, \\
\theta_{j} I_{N}-\frac{\xi}{\lambda_{\max }(P)} I_{N}+c \gamma_{j} A \leq 0, \quad j=1,2, \ldots, n,
\end{array}
$$

where $\Gamma=\operatorname{diag}\left(\gamma_{1}, \gamma_{2}, \ldots, \gamma_{n}\right), \Xi=\operatorname{diag}\left(\eta_{1}, \eta_{2}, \ldots, \eta_{n}\right), \Theta=$ $\operatorname{diag}\left(\theta_{1}, \theta_{2}, \ldots, \theta_{n}\right)$, and $I_{N}$ is the $N \times N$ identity matrix. Then under the periodically intermittent controllers (37), the error system (38) is synchronized in a finite time:

$$
T_{1}=-\frac{2 \ln \left(1-(\beta / \sqrt{2} \bar{k}) V^{1 / 2}(0)\right)}{\theta \beta},
$$

where $V(0)=(1 / 2) \sum_{i=1}^{N} e_{i}^{T}(0) P e_{i}(0)$ and $e_{i}(0)$ is the initial condition of $e_{i}(t)$.

Proof. Consider the following Lyapunov function:

$$
V(t)=\frac{1}{2} \sum_{i=1}^{N} e_{i}^{T}(t) P e_{i}(t)
$$


Then the time derivative of (42) along the trajectories of the first subsystem of system (38) is calculated and estimated as follows.

When $l T \leq t<(l+\theta) T$, for $l \in l$,

$$
\begin{aligned}
& \dot{V}(t)=\sum_{i=1}^{N} e_{i}^{T}(t) P \dot{e}_{i}(t)=\sum_{i=1}^{N} e_{i}^{T}(t) P\left[f\left(y_{i}(t)\right)\right. \\
& \left.-f\left(x_{i}(t)\right)+c \sum_{j=1}^{N} a_{i j} \Gamma e_{j}(t)+u_{i}(t)\right] \\
& \quad=\sum_{i=1}^{N}\left\{e_{i}^{T}(t) P\left[f\left(y_{i}(t)\right)-f\left(x_{i}(t)\right)-\Theta e_{i}(t)\right]\right.
\end{aligned}
$$$$
+e_{i}^{T}(t) P \Theta e_{i}(t)+e_{i}^{T}(t) P u_{i}(t)
$$$$
\left.+c e_{i}^{T}(t) P \Gamma \sum_{j=1}^{N} a_{i j} e_{j}(t)\right\} \leq-\xi \sum_{i=1}^{N} e_{i}^{T}(t) e_{i}(t)
$$$$
+\sum_{i=1}^{N} e_{i}^{T}(t)\left(\Theta P-\eta_{i} P\right) e_{i}(t)+c \sum_{i=1}^{N} e_{i}^{T} \Gamma \sum_{j=1}^{N} a_{i j} P e_{j}(t)
$$$$
-\bar{k} \sum_{i=1}^{N} \frac{\sqrt{\lambda_{\max }(P)}}{\lambda_{\min }(P)} e_{i}^{T}(t) P \operatorname{sign}\left(e_{i}\right)-\beta \sum_{i=1}^{N} e_{i}^{T}(t)
$$$$
\cdot e_{i}(t)+\beta \sum_{i=1}^{N} e_{i}^{T}(t) e_{i}(t) \leq \sum_{j=1}^{n} p_{j} \tilde{e}_{j}^{T}(t)\left(\theta_{j} I_{N}-\Xi\right.
$$$$
\left.+c \gamma_{j} A-\beta I_{N}\right) \tilde{e}_{j}(t)+\beta \sum_{i=1}^{N} e_{i}^{T}(t) e_{i}(t)
$$$$
-\bar{k} \sum_{i=1}^{N} \frac{\sqrt{\lambda_{\max }(P)}}{\lambda_{\min }(P)} e_{i}^{T}(t) P \operatorname{sign}\left(e_{i}\right)=\sum_{j=1}^{n} p_{j} \widetilde{e}_{j}^{T}(t)
$$$$
\cdot\left(\theta_{j} I_{N}-\Xi+c \gamma_{j} A-\beta I_{N}\right) \tilde{e}_{j}(t)+\beta \sum_{i=1}^{N} e_{i}^{T}(t) e_{i}(t)
$$$$
-\bar{k} \sum_{i=1}^{N} \frac{\sqrt{\lambda_{\max }(P)}}{\lambda_{\min }(P)} e_{i}^{T}(t) P \operatorname{sign}\left(e_{i}\right) .
$$

Defining $\operatorname{sign}\left(e_{i}\right)=\left(\operatorname{sign}\left(e_{i 1}\right), \operatorname{sign}\left(e_{i 2}\right), \ldots, \operatorname{sign}\left(e_{i n}\right)\right)^{T}$, we have

$$
\begin{aligned}
\dot{V}(t) \leq & \beta \sum_{i=1}^{N} e_{i}^{T}(t) P e_{i}(t)+\sum_{j=1}^{n} p_{j} \widetilde{e}_{j}^{T}(t) Z_{j} \widetilde{e}_{j}^{T}(t) \\
& -\bar{k} \sum_{i=1}^{N} \sqrt{\lambda_{\max }(P)}\left\|e_{i}\right\|,
\end{aligned}
$$

where $\widetilde{e}_{j}(t)=\left[\tilde{e}_{j 1}, \widetilde{e}_{j 2}, \ldots, \widetilde{e}_{j N}\right]$ is a column vector of $e_{j}(t)$ and $Z_{j}$ is defined as

$$
Z_{j}=\theta_{j} I_{N}-\Xi+c \gamma_{j} A-\beta I_{N}, \quad j=1,2, \ldots, n .
$$

Using Lemma 7, we have

$$
\begin{aligned}
\dot{V}(t) \leq & \beta \sum_{i=1}^{N} e_{i}^{T}(t) P e_{i}(t)+\sum_{j=1}^{n} p_{j} \tilde{e}_{j}^{T}(t) Z_{j} \tilde{e}_{j}^{T}(t) \\
& -\sqrt{2} \bar{k}\left(\frac{1}{2} \sum_{i=1}^{N} e_{i}^{T}(t) P e_{i}(t)\right)^{1 / 2} .
\end{aligned}
$$

It follows from inequality (39) that

$$
Z_{j} \leq 0
$$

which shows that $\dot{V}(t) \leq-\sqrt{2} \bar{k} V^{1 / 2}(t)+\beta V(t)$.

When $(l+\theta) T \leq t<(l+1) T$, for $l \in l$, we have

$$
\begin{aligned}
\dot{V}(t) & =\sum_{i=1}^{N} e_{i}^{T}(t) P \dot{e}_{i}(t) \\
& =\sum_{i=1}^{N}\left\{e_{i}^{T}(t) P\left[f\left(y_{i}(t)\right)-f\left(x_{i}(t)\right)-\Theta e_{i}(t)\right]\right. \\
& \left.+e_{i}^{T}(t) P \Theta e_{i}(t)+c \sum_{j=1}^{N} a_{i j} e_{i}^{T}(t) P \Gamma e_{j}(t)\right\} \\
& \leq-\xi \sum_{i=1}^{N} e_{i}^{T}(t) e_{i}(t)+\sum_{j=1}^{n} p_{j} \widetilde{e}_{j}^{T}(t) \theta_{j} I_{N} \widetilde{e}_{j}(t) \\
& +c \sum_{j=1}^{n} p_{j} \tilde{e}_{j}^{T}(t) \gamma_{j} A \widetilde{e}_{j}(t) \leq-\sum_{j=1}^{n} p_{j} \widetilde{e}_{j}^{T}(t) \\
& \cdot \frac{\xi}{\lambda_{\max }(P)} I_{N} \widetilde{e}_{j}(t)+\sum_{j=1}^{n} p_{j} \tilde{e}_{j}^{T}(t) \theta_{j} I_{N} \widetilde{e}_{j}(t) \\
& +c \sum_{j=1}^{n} p_{j} \widetilde{e}_{j}^{T}(t) \gamma_{j} A \widetilde{e}_{j}(t)=\sum_{j=1}^{n} p_{j} \widetilde{e}_{j}^{T}(t) S_{j} \widetilde{e}_{j}^{T}(t),
\end{aligned}
$$

where $\widetilde{e}_{j}(t)=\left[\widetilde{e}_{j 1}, \widetilde{e}_{j 2}, \ldots, \widetilde{e}_{j N}\right]$ is a column vector of $e_{j}(t)$ and $S_{j}$ is defined as

$$
S_{j}=\theta_{j} I_{N}-\frac{\xi}{\lambda_{\max }(P)} I_{N}+c \gamma_{j} A, \quad j=1,2, \ldots, n .
$$

It follows from inequality (40) that

$$
S_{j} \leq 0
$$

which shows that $\dot{V}(t) \leq 0$. 
Namely, defining $\alpha=\sqrt{2} \bar{k}, \eta=1 / 2$, and $p=\beta$, we get

$$
\begin{aligned}
& D^{+} V(t) \leq-\alpha V^{\eta}(t)+p V(t), \quad l T \leq t \leq l T+\theta T, \\
& D^{+} V(t) \leq 0, \quad l T+\theta T \leq t<(l+1) T .
\end{aligned}
$$

According to Lemma 5, we have

$$
\begin{aligned}
& V^{1-\eta}(t) \exp \{-(1-\eta) p t\} \\
& \quad \leq V^{1-\eta}(0)+\frac{\alpha}{p}[\exp \{-(1-\eta) p \theta t\}-1], \\
& \qquad 0 \leq t \leq T_{1} .
\end{aligned}
$$

By Lemma 3, we have

$$
\begin{aligned}
t & \leq \frac{\ln \left(1-(p / \alpha) V^{1-\eta}(0)\right)}{p \theta(\eta-1)} \\
& =-\frac{2 \ln \left(1-(\beta / \sqrt{2} \bar{k}) V^{1 / 2}(0)\right)}{\theta \beta}, \quad 0 \leq t \leq T_{1} .
\end{aligned}
$$

The proof of Theorem 8 is completed.

Remark 9. Obviously, when $\theta=1$, the intermittent control (37) is degenerated to a continuous control input which has been extensively proposed in previous work (see [34, 35]) and focuses on [13]. However, this trivial case is not to be discussed in this paper.

If the Lyapunov function $\dot{V}(t) \leq-\alpha V(t)(p=0)$ when controllers are added into the network, then it is easy to see that Theorem 8 can be restated as the following corollary.

Corollary 10. Let Assumption 1 hold. Suppose that positive constants $\eta_{1}, \eta_{2}, \ldots, \eta_{N}, \xi$, and a positive define diagonal matrix $P>0$ satisfy

$$
\begin{array}{r}
\theta_{j} I_{N}-\Xi+c \gamma_{j} A \leq 0, \quad j=1,2, \ldots, n, \\
\theta_{j} I_{N}-\frac{\xi}{\lambda_{\max }(P)} I_{N}+c \gamma_{j} A \leq 0, \quad j=1,2, \ldots, n,
\end{array}
$$

where $\Gamma=\operatorname{diag}\left(\gamma_{1}, \gamma_{2}, \ldots, \gamma_{n}\right), \Xi=\operatorname{diag}\left(\eta_{1}, \eta_{2}, \ldots, \eta_{n}\right), \Theta=$ $\operatorname{diag}\left(\theta_{1}, \theta_{2}, \ldots, \theta_{n}\right)$, and $I_{N}$ is the $N \times N$ identity matrix. Then under the periodically intermittent controllers (37), the error system (38) is synchronized in a finite time:

$$
T_{2}=\frac{V^{1-\eta}(0)}{\sqrt{2} \bar{k} \theta}
$$

where $V(0)=(1 / 2) \sum_{i=1}^{N} e_{i}^{T}(0) P e_{i}(0)$ and $e_{i}(0)$ is the initial condition of $e_{i}(t)$.

Remark 11. Corollary 10 in this paper is the main result of Theorem 2 in [30] and the main results in [36].

Remark 12. According to (41) and (56) and the convergence time $T_{1}, T_{2}$, we can conclude that the convergence time satisfies $T_{2} \leq T_{1}$. We can analyse that the term $\beta V(t)$ should impede the convergence time. But compared with the control gain matrix $\Xi$ in (54), it is easy to seek an appropriate control gain matrix $\Xi$ in (39) for which synchronization happens.

Remark 13. It is clear to see that inequality (39) is more easily satisfied compared with inequality (54) under the same controllers and conditions via LMI Toolbox, which reveals a very interesting phenomenon; that is, the control gain under condition (39) is more easily designed than condition (54), though it can impede the convergence time.

Remark 14. We can find that if condition (55) is satisfied, condition (54) easily holds when the positive definite control gain matrix $\Xi$ is anything. Then we have the following corollary.

Corollary 15. Let Assumption 1 hold. Suppose that positive constants $\eta_{1}, \eta_{2}, \ldots, \eta_{N}, \xi$, and a positive defined diagonal matrix $P>0$ satisfy

$$
\theta_{j} I_{N}-\frac{\xi}{\lambda_{\max }(P)} I_{N}+c \gamma_{j} A \leq 0, \quad j=1,2, \ldots, n,
$$

where $\Gamma=\operatorname{diag}\left(\gamma_{1}, \gamma_{2}, \ldots, \gamma_{n}\right), \Theta=\operatorname{diag}\left(\theta_{1}, \theta_{2}, \ldots, \theta_{n}\right)$, and $I_{N}$ is the $N \times N$ identity matrix. Then under the periodically intermittent controllers (37), the error system (38) is synchronized in a finite time:

$$
T_{3}=\frac{V^{1-\eta}(0)}{\sqrt{2} \bar{k} \theta}
$$

where $V(0)=(1 / 2) \sum_{i=1}^{N} e_{i}^{T}(0) P e_{i}(0)$ and $e_{i}(0)$ is the initial condition of $e_{i}(t)$.

\section{Numerical Examples}

In this section, we give some numerical examples to show the validity and effectiveness of the derived results for finite-time synchronization via periodically intermittent control.

In this case, to demonstrate the results above, we consider general complex dynamical networks, in which each subsystem is a Lorenz system. The dynamics of Lorenz system is described as follows:

$$
\begin{aligned}
\dot{s} & =f(s)=\left[\begin{array}{c}
\dot{s}_{1} \\
\dot{s}_{2} \\
\dot{s}_{3}
\end{array}\right]=\left[\begin{array}{ccc}
-a & a & 0 \\
c & -1 & 0 \\
0 & 0 & -b
\end{array}\right]\left[\begin{array}{l}
s_{1} \\
s_{2} \\
s_{3}
\end{array}\right]+\left[\begin{array}{c}
0 \\
-s_{1} s_{3} \\
s_{1} s_{2}
\end{array}\right] \\
& \triangleq C s+\psi(s),
\end{aligned}
$$

where the parameters are selected as $a=10, c=30$, and $b=8 / 3$; then the Lorenz system has a chaotic attractor (see Figure 1). Moreover, it is known that $\left|x_{i 1}\right| \leq 18.6360=r_{1}$, $\left|x_{i 2}\right| \leq 25.3679=r_{2}$, and $\left|x_{i 3}\right| \leq 45.9792=r_{3}$. Now we will show that there exists a positive definite diagonal matrix $P$ that satisfies Assumption 1. Let $x=\left[x_{1}, x_{2}, x_{3}\right]^{T}$, 




FIGURE 1: The chaotic trajectories of the Lorenz system.

$y=\left[y_{1}, y_{2}, y_{3}\right]^{T}, \delta=x-y=\left[\delta_{1}, \delta_{2}, \delta_{3}\right]^{T}=\left[x_{1}-y_{1}, x_{2}-\right.$ $\left.y_{2}, x_{3}-y_{3}\right]^{T}$, and $|\epsilon|=\left[\left|\epsilon_{1}\right|,\left|\epsilon_{2}\right|,\left|\epsilon_{3}\right|\right]^{T}$; then

$$
\begin{aligned}
\psi(x)-\psi(y) & =\left[\begin{array}{c}
0 \\
-x_{1} x_{3}+y_{1} y_{3} \\
x_{1} x_{2}-y_{1} y_{2}
\end{array}\right] \\
& =\left[\begin{array}{c}
0 \\
-x_{1} \epsilon_{3}-y_{3} \epsilon_{1} \\
x_{1} \epsilon_{2}+y_{2} \epsilon_{1}
\end{array}\right] .
\end{aligned}
$$

Hence,

$$
\begin{aligned}
& (x-y)^{T}(\psi(x)-\psi(y)) \\
& =-y_{3} \epsilon_{1} \epsilon_{2}+y_{2} \epsilon_{1} \epsilon_{3} \leq \frac{1}{2}|\epsilon|^{T}\left[\begin{array}{ccc}
0 & r_{3} & r_{2} \\
r_{3} & 0 & 0 \\
r_{2} & 0 & 0
\end{array}\right]|\epsilon| \\
& \triangleq \frac{1}{2}|\epsilon|^{T} R|\epsilon| .
\end{aligned}
$$

Then

$$
\begin{aligned}
& (x-y)^{T} P(f(x)-f(y)-\Theta(x-y)) \\
& \leq \frac{\lambda_{\max }(C)+(1 / 2) \lambda_{\max }(R)-\lambda_{\min }(\Theta)}{\lambda_{\min }(P)}(x-y)^{T} \\
& \cdot(x-y),
\end{aligned}
$$

where $\lambda_{\max }(C), \lambda_{\max }(R), \lambda_{\min }(\Theta)$ are the maximum eigenvalues of $C, R, \Theta$, respectively. Let $\xi=-\left(\lambda_{\max }(C)+\right.$ $\left.(1 / 2) \lambda_{\text {max }}(R)-\lambda_{\text {min }}(\Theta)\right) / \lambda_{\text {min }}(P)=43.48$; therefore Assumption 1 is satisfied with $\Theta=\operatorname{diag}(50,50,50)$ and $P=$ $\operatorname{diag}(0.5,0.4,0.2)$.

Consider the complex dynamical network (1) consisting of 50 identical Lorenz oscillators nodes, which is described by

$$
\dot{x}_{i}(t)=f\left(x_{i}(t)\right)+c \sum_{j=1}^{50} a_{i j} \Gamma x_{j}(t), \quad i=1,2, \ldots, 50,
$$

where $\Gamma=\operatorname{diag}(1,1,1)$ and $A=\left(a_{i j}\right)_{50 \times 50}$ is a symmetrically diffusive coupling matrix with $a_{i j}=0$ or $1(j \neq i)$ and the coupling strength $c=1$.

The corresponding controlled response system of (36) is of the form

$$
\begin{aligned}
\dot{y}_{i}(t)=f\left(y_{i}(t)\right)+c \sum_{j=1}^{50} a_{i j} \Gamma y_{j}(t)+u_{i}(t) & \\
& \\
i & =1,2, \ldots, 50
\end{aligned}
$$

where $a_{i j}$ and $f_{i}(\cdot)$ are the same as $(63)$ and

$$
\begin{aligned}
& u_{i}(t)=-\eta_{i} e_{i}(t)-\bar{k} \frac{\sqrt{\lambda_{\max }(P)}}{\lambda_{\min }(P)} \operatorname{sign}\left(e_{i}\right), \\
& \quad l T \leq t<l T+\delta, \\
& u_{i}(t)=0, \quad\left\|e_{i}\right\|=0 \text { or } l T+\delta \leq t<(l+1) T,
\end{aligned}
$$

where $\bar{k}=5$.

The initial states of the numerical simulations in the master and slave systems are as follows: $x_{i}(0)=(2+0.2 i, 0.2+$ $0.3 i, 0.3+0.1 i)^{T}$ and $y_{i}(0)=(-8+0.7 i,-5+0.8 i,-10+0.6 i)^{T}$. The initial conditions of the error system are $e_{i}(0)=(-10+$ $0.5 i,-5.2+0.5 i,-10.3+0.5 i)^{T}$, where $1 \leq i \leq 50$. In addition, the values of the parameters for the controllers (37) are selected as $T=1$ and $\delta=0.8$. Choosing $\Theta=\operatorname{diag}(50,50,50)$, $P=\operatorname{diag}(0.5,0.4,0.2)$, and $\lambda_{\text {max }}(A)=-1.6414$, one can easily obtain that inequality (40) holds. Besides, we can obtain the parameters of the intermittent controllers as $\Xi=$ $\operatorname{diag}[57.9071, \ldots, 57.9071]_{50 \times 50}$ and $\beta=1.304$ by using LMI toolbox in Matlab to solve (39) and (40). Therefore from Theorem 8 it can be obtained that the response system (36) will synchronize the drive system (1) under the periodically intermittent controllers (37) within the time $T=1.234$ (the average time). The synchronous error $e_{i}(t)$ is illustrated in Figures 2-4.

Take $\bar{k}=5, T=0.5$, and $\theta=0.4$. By calculating (41), we can obtain the convergence time $t=2.242$. The time responses of the error variables $e_{i j}, 1 \leq i \leq 50, j=1,2,3$, are illustrated in Figures 5-7, with $\bar{k}=5, T=0.5$, and $\theta=0.4$. Let $\bar{k}=5, T=0.5$, and $\theta=0.1$. By calculating (41), we can obtain the convergence time $t=3.278$. Figures $8-10$ describe the time responses or the error variables $e_{i j}, 1 \leq i \leq 50, j=1,2,3$, respectively, with different parameters of $\bar{k}, T, \theta$.

Remark 16. From the above analysis and figures, we can conclude that the control rate influences the convergence time; in addition, the tunable $\bar{k}$ can also influence the convergence time, which is discussed in [34]; we should omit it here. And then, it can be seen that the continuous controller can synchronize the network at $t=0.984$. Therefore, the convergence time is shorter than the periodically intermittent control.

To further verify the effectiveness of the proposed control design, we take the control rate $\theta=1$, namely, general continuous full control. Figures 11-13 demonstrate time response of 


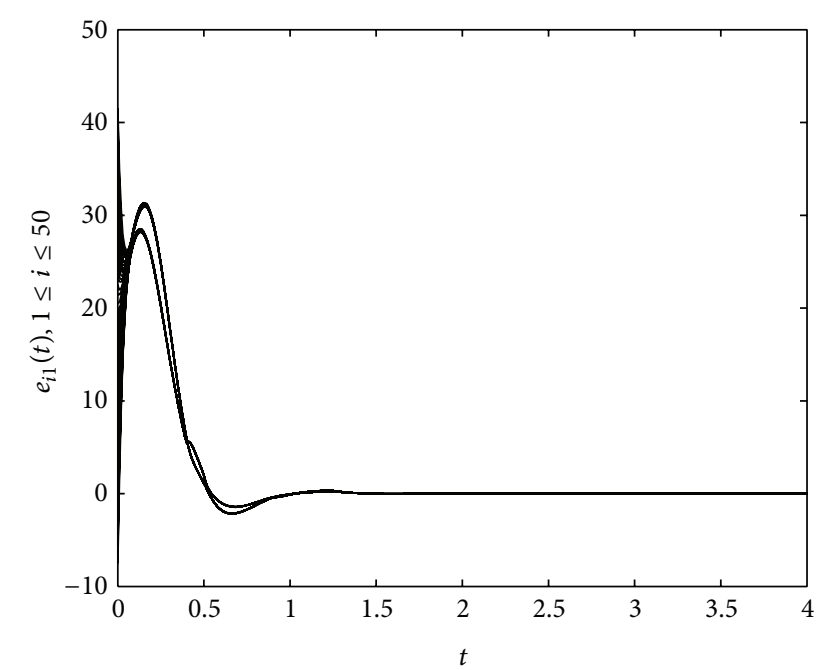

FIGURE 2: Synchronization errors $e_{i 1}, 1 \leq i \leq 50$, with control parameters $\bar{k}=5, T=0.5$, and $\theta=0.8$.

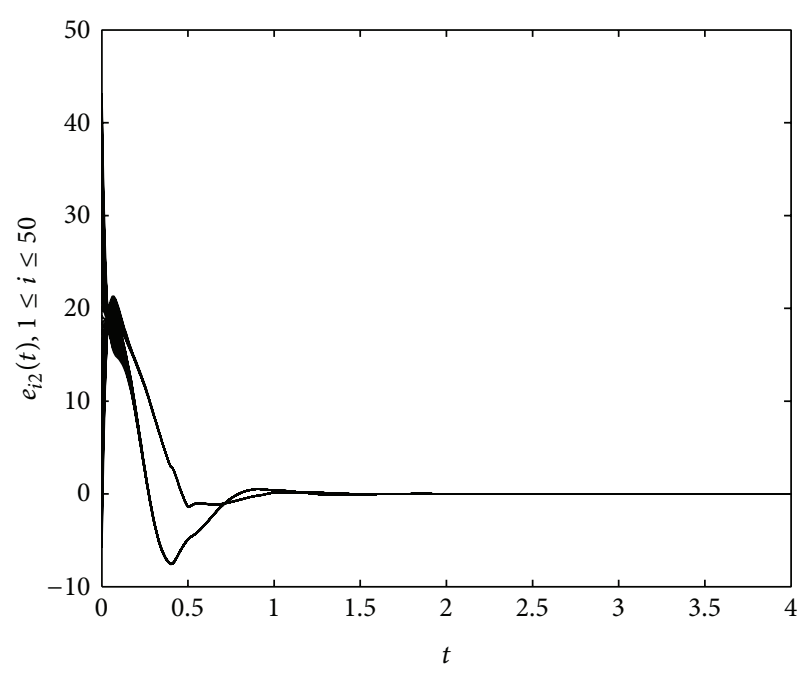

FIGURE 3: Synchronization errors $e_{i 2}, 1 \leq i \leq 50$, with control parameters $\bar{k}=5, T=0.5$, and $\theta=0.8$.

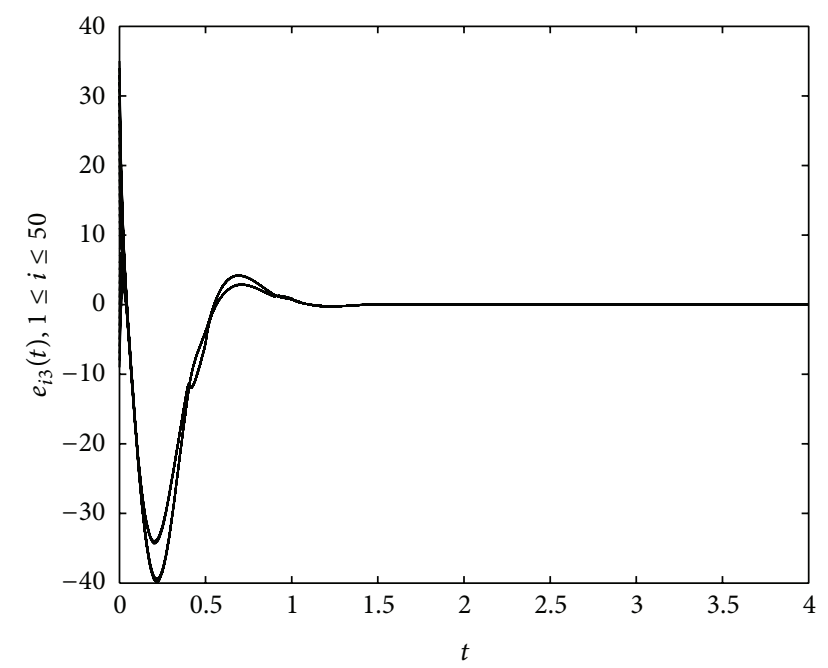

FIGURE 4: Synchronization errors $e_{i 3}, 1 \leq i \leq 50$, with control parameters $\bar{k}=5, T=0.5$, and $\theta=0.8$.

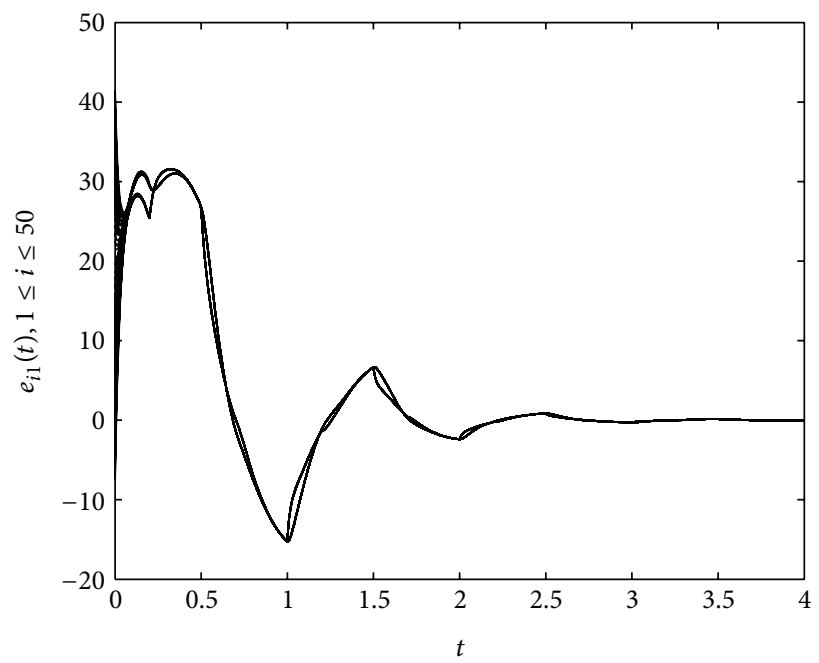

FIGURE 5: Synchronization errors $e_{i 1}, 1 \leq i \leq 50$, with control parameters $\bar{k}=5, T=0.5$, and $\theta=0.4$.

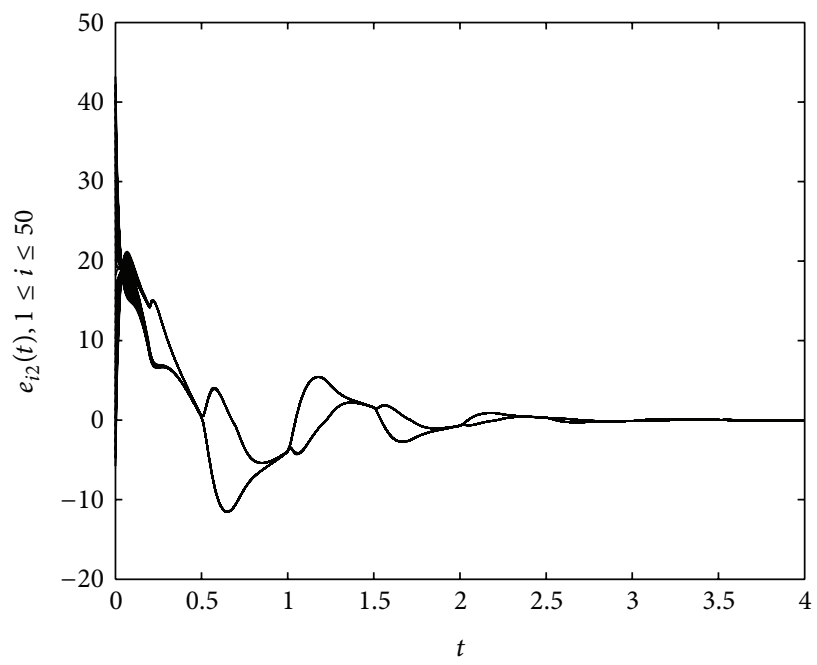

FIGURE 6: Synchronization errors $e_{i 2}, 1 \leq i \leq 50$, with control parameters $\bar{k}=5, T=0.5$, and $\theta=0.4$.

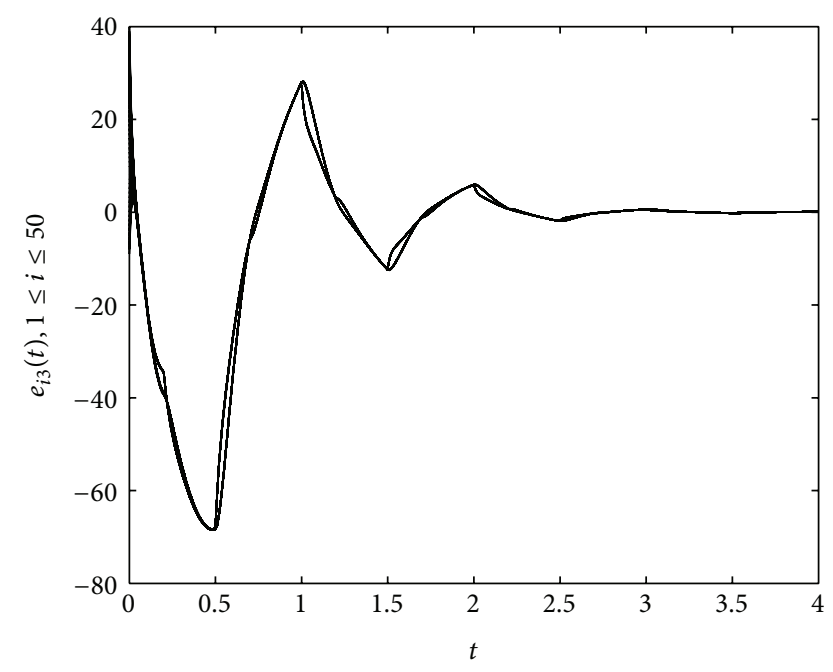

FIgURE 7: Synchronization errors $e_{i 3}, 1 \leq i \leq 50$, with control parameters $\bar{k}=5, T=0.5$, and $\theta=0.4$. 


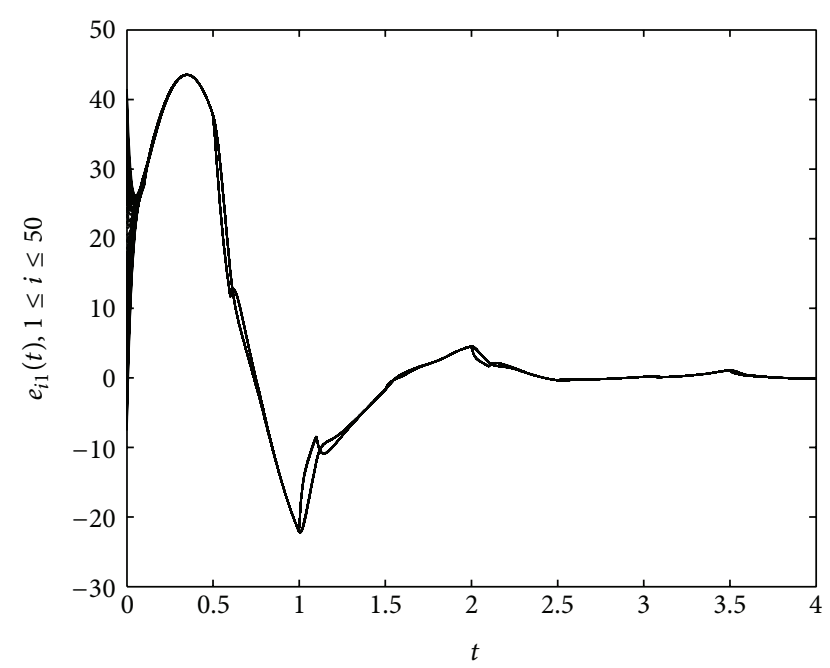

FIGURE 8: Synchronization errors $e_{i 1}, 1 \leq i \leq 50$, with control parameters $\bar{k}=5, T=0.5$, and $\theta=0.1$.

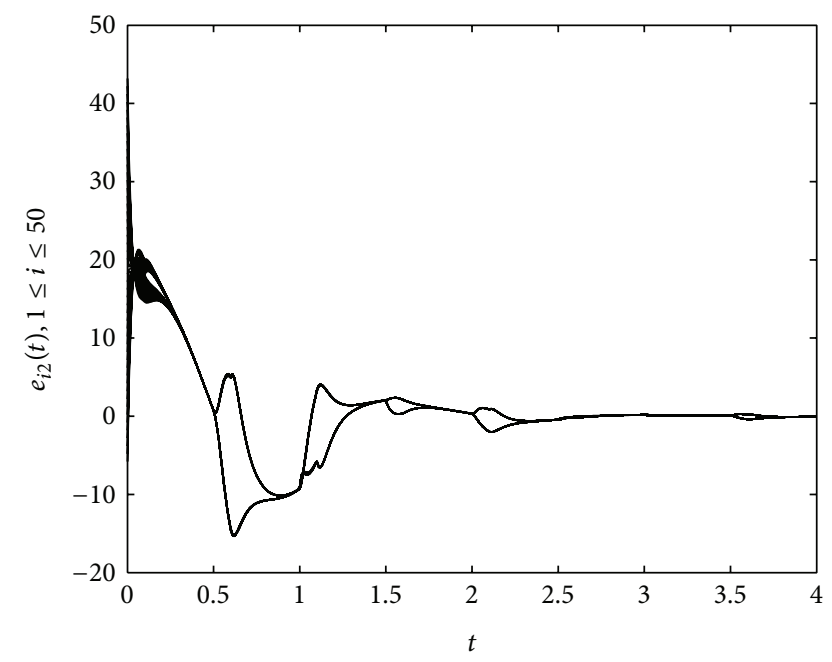

FIGURE 9: Synchronization errors $e_{i 2}, 1 \leq i \leq 50$, with control parameters $\bar{k}=5, T=0.5$, and $\theta=0.1$.

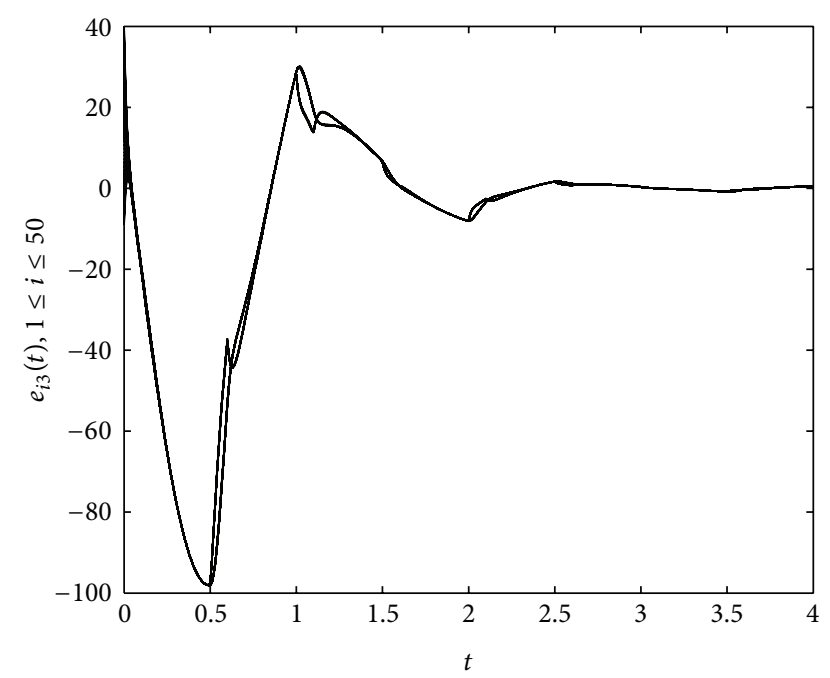

FIGURE 10: Synchronization errors $e_{i 3}, 1 \leq i \leq 50$, with control parameters $\bar{k}=5, T=0.5$, and $\theta=0.1$.

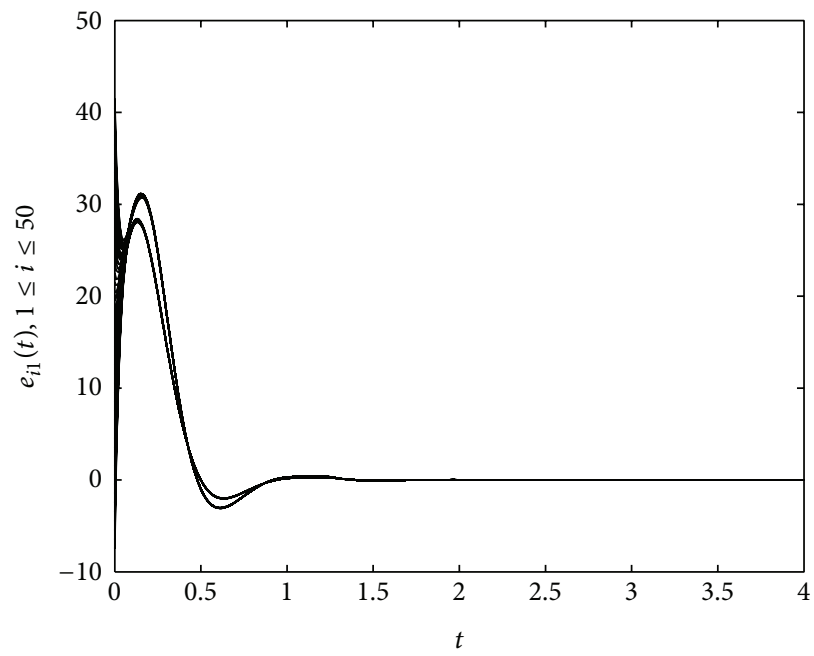

FIGURE 11: Synchronization errors $e_{i 1}, 1 \leq i \leq 50$, with full control parameters $\bar{k}=5, T=0.5$, and $\theta=1$.

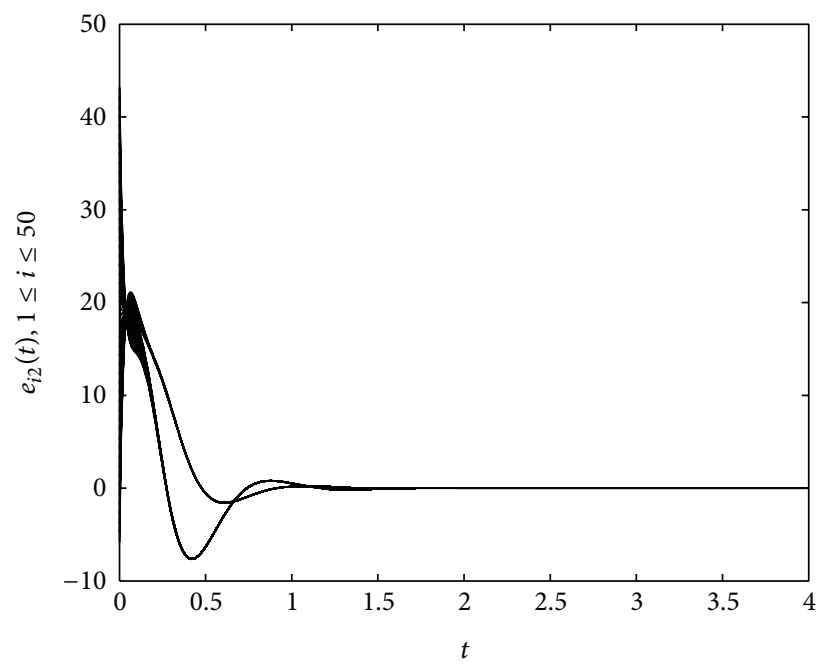

FIGURE 12: Synchronization errors $e_{i 2}, 1 \leq i \leq 50$, with full control parameters $\bar{k}=5, T=0.5$, and $\theta=1$.

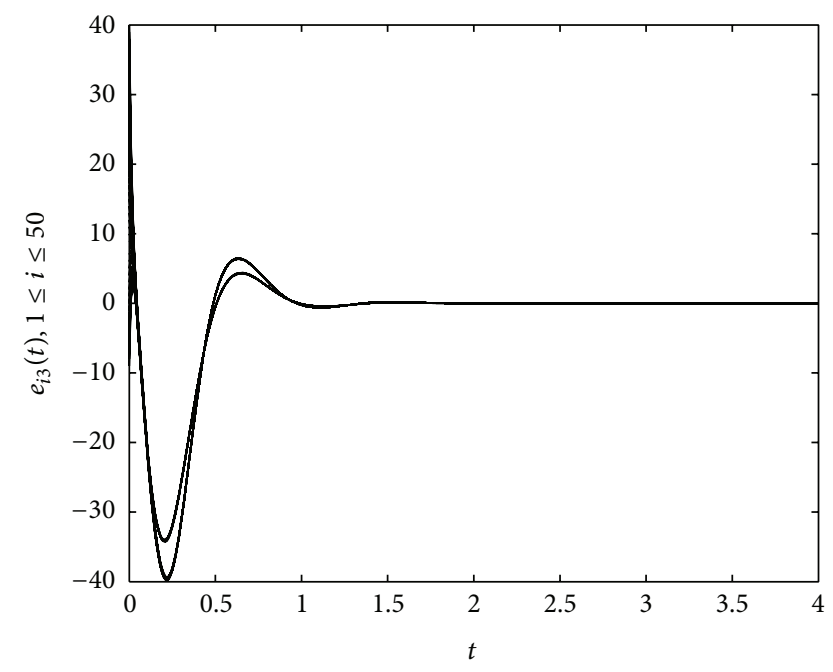

FIGURE 13: Synchronization errors $e_{i 3}, 1 \leq i \leq 50$, with full control parameters $\bar{k}=5, T=0.5$, and $\theta=1$. 


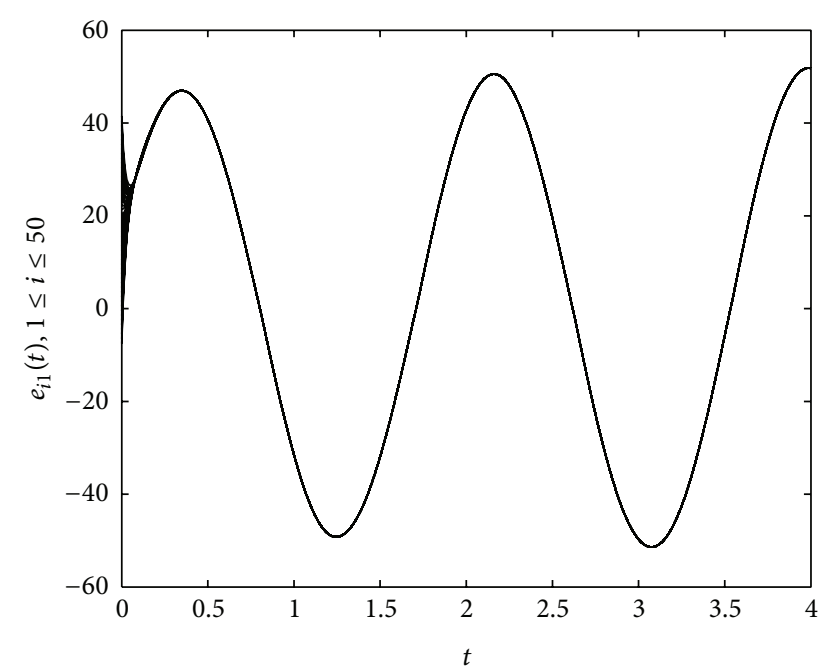

FIGURE 14: Synchronization errors $e_{i 1}, 1 \leq i \leq 50$, with no control input.

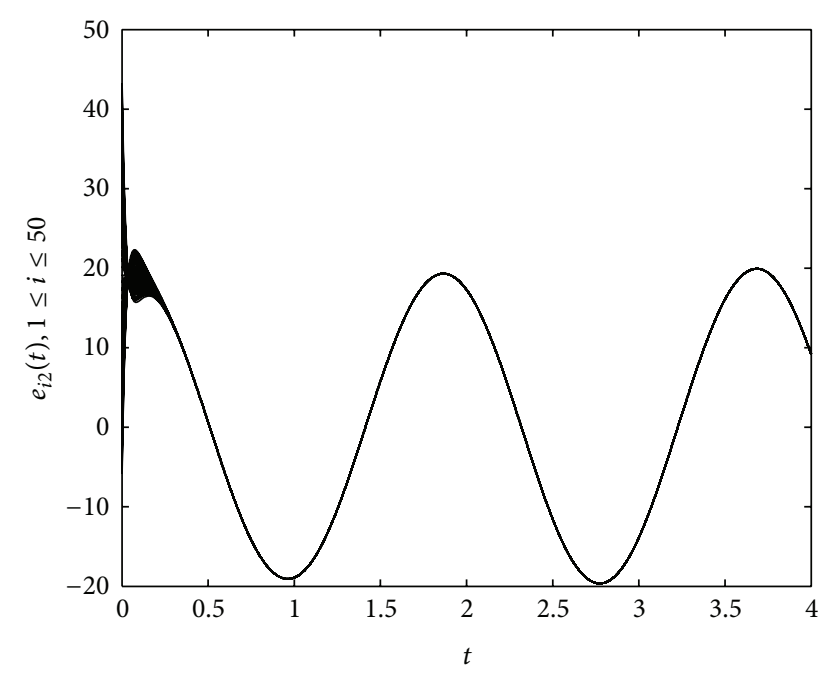

FIGURE 15: Synchronization errors $e_{i 2}, 1 \leq i \leq 50$, with no control input.

the error variables $e_{i j}, 1 \leq i \leq 50, j=1,2,3$, respectively, with $\bar{k}=5, T=0.5$, and $\theta=1$. When the control variables $u_{i}=0$, $1 \leq i \leq 50$, namely no controlled is added to the system. The time response of the error variables $e_{i j}, 1 \leq i \leq 50$, $j=1,2,3$, is displayed in Figures 14-16, respectively, with no control input $u_{i}$.

\section{Conclusion}

This paper has dealt with the finite-time synchronization problem for a class of complex dynamical networks by means of periodically intermittent control. Some novel and useful synchronization criteria, given in terms of a set of linear matrix inequalities, have been obtained by some analysis techniques and finite-time stability theory. Our results reduce the previous works on the controllers that the derivative of

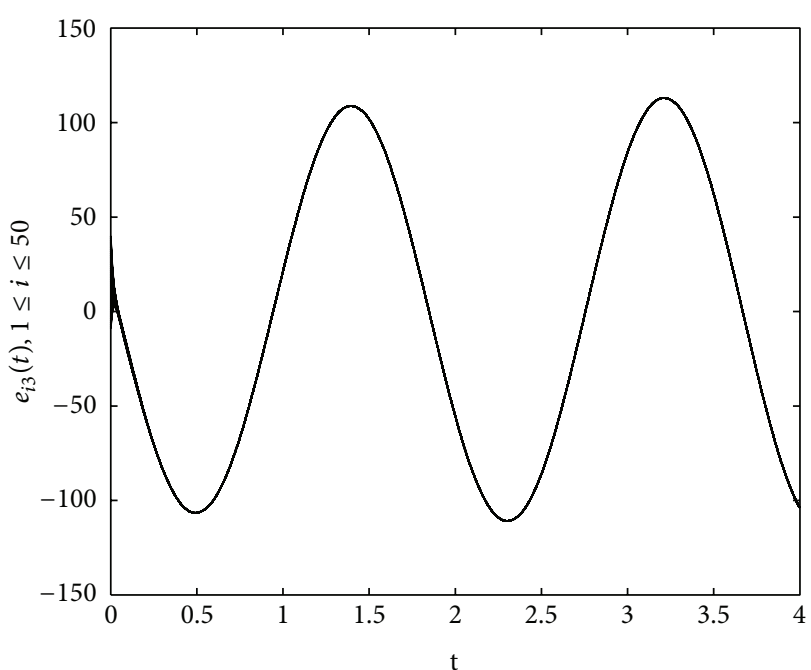

FIGURE 16: Synchronization errors $e_{i 3}, 1 \leq i \leq 50$, with no control input.

the Lyapunov function $V(t)$ is smaller than $-\alpha V^{\eta}(t)$. Several simulations are presented to verify the effectiveness of the proposed synchronization criteria finally. The future work in this endeavor will focus on the global problem.

\section{Conflict of Interests}

The authors declare that there is no conflict of interests regarding the publication of this paper.

\section{Acknowledgments}

The authors would like to thank the editor and the anonymous reviewers for their valuable comments and constructive suggestions. This project is supported by the National Science Foundation of China (11371162 and 1171129) and the Education Department of Hubei Province (T201103).

\section{References}

[1] S. Boccaletti, V. Latora, Y. Moreno, M. Chavez, and D.-U. Hwang, "Complex networks: structure and dynamics," Physics Reports A, vol. 424, no. 4-5, pp. 175-308, 2006.

[2] A. Arenas, A. Díaz-Guilera, J. Kurths, Y. Moreno, and C. Zhou, "Synchronization in complex networks," Physics Reports, vol. 469, no. 3, pp. 93-153, 2008.

[3] A. Pikovsky, M. Rosenblum, and J. Kurths, Synchronization: A Universal Concept in Nonlinear Sciences, vol. 12 of Cambridge Nonlinear Science Series, Cambridge University Press, Cambridge, UK, 2001.

[4] S. H. Strogatz, "Exploring complex networks," Nature, vol. 410, no. 6825, pp. 268-276, 2001.

[5] X. F. Wang and G. Chen, "Synchronization in small-world dynamical networks," International Journal of Bifurcation and Chaos, vol. 12, no. 1, pp. 187-192, 2002.

[6] C. Wu, Synchronization in Complex Networks of Nonlinear Dynamical Systems, World Scientific, Singapore, 2007. 
[7] X. Wang and G. Chen, "Synchronization in scale-free dynamical networks: robustness and fragility," IEEE Transactions on Circuits and Systems I: Fundamental Theory and Applications, vol. 49, no. 1, pp. 54-62, 2002.

[8] J. Mei, M. Jiang, and Z. Huang, "Outer synchronization between two complex networks with identical and nonidentical topological structures," in Proceedings of the 4th International Workshop on Advanced Computational Intelligence (IWACI '11), pp. 757762, October 2011.

[9] H. Yu and X. Xia, "Adaptive consensus of multi-agents in networks with jointly connected topologies," Automatica, vol. 48, no. 8, pp. 1783-1790, 2012.

[10] H. Yu, Y. Shen, and X. Xia, "Adaptive finite-time consensus in multi-agent networks," Systems \& Control Letters, vol. 62, no. 10, pp. 880-889, 2013.

[11] M. Jiang, S. Wang, J. Mei, and Y. Shen, "Finite-time synchronization control of a class of memristor-based recurrent neural networks," Neural Networks, vol. 63, pp. 133-140, 2015.

[12] M. Jiang, J. Mei, and J. Hu, "New results on exponential synchronization of memristor-based chaotic neural networks," Neurocomputing, vol. 156, pp. 60-67, 2015.

[13] Y. Shen, Y. Huang, and J. Gu, "Global finite-time observers for lipschitz nonlinear systems," IEEE Transactions on Automatic Control, vol. 56, no. 2, pp. 418-424, 2011.

[14] Y. Shen and Y. Huang, "Uniformly observable and globally Lipschitzian nonlinear systems admit global finite-time observers," IEEE Transactions on Automatic Control, vol. 54, no. 11, pp. 2621-2625, 2009.

[15] X. Yang, J. Cao, and J. Lu, "Synchronization of delayed complex dynamical networks with impulsive and stochastic effects," Nonlinear Analysis: Real World Applications, vol. 12, no. 4, pp. 2252-2266, 2011.

[16] Q. Zhang, J.-a. Lu, and J. Zhao, "Impulsive synchronization of general continuous and discrete-time complex dynamical networks," Communications in Nonlinear Science and Numerical Simulation, vol. 15, no. 4, pp. 1063-1070, 2010.

[17] M. Sun, C. Zeng, Y. Tao, and L. Tian, "Adaptive-impulsive synchronization in drive-response networks of continuous systems and its application," Physics Letters. Section A. General, Atomic and Solid State Physics, vol. 373, no. 34, pp. 3041-3046, 2009.

[18] S. Zheng, G. Dong, and Q. Bi, "Impulsive synchronization of complex networks with non-delayed and delayed coupling," Physics Letters, Section A: General, Atomic and Solid State Physics, vol. 373, no. 46, pp. 4255-4259, 2009.

[19] S. Cai, J. Zhou, L. Xiang, and Z. Liu, "Robust impulsive synchronization of complex delayed dynamical networks," Physics Letters A, vol. 372, no. 30, pp. 4990-4995, 2008.

[20] W. Xia and J. Cao, "Pinning synchronization of delayed dynamical networks via periodically intermittent control," Chaos, vol. 19, no. 1, Article ID 013120, 2009.

[21] S. Cai, Z. Liu, F. Xu, and J. Shen, "Periodically intermittent controlling complex dynamical networks with time-varying delays to a desired orbit," Physics Letters A, vol. 373, no. 42, pp. 3846-3854, 2009.

[22] S. Cai, J. Hao, Q. He, and Z. Liu, "Exponential synchronization of complex delayed dynamical networks via pinning periodically intermittent control," Physics Letters A, vol. 375, no. 19, pp. 1965-1971, 2011.
[23] J. Mei, M. Jiang, Z. Wu, and X. Wang, "Periodically intermittent controlling for finite-time synchronization of complex dynamical networks," Nonlinear Dynamics, vol. 79, no. 1, pp. 295-305, 2015.

[24] J. Yu, C. Hu, H. Jiang, and Z. Teng, "Synchronization of nonlinear systems with delays via periodically nonlinear intermittent control," Communications in Nonlinear Science and Numerical Simulation, vol. 17, no. 7, pp. 2978-2989, 2012.

[25] H. Zhu and B. Cui, "Stabilization and synchronization of chaotic systems via intermittent control," Communications in Nonlinear Science and Numerical Simulation, vol. 15, no. 11, pp. 3577-3586, 2010.

[26] J. Mei, M. Jiang, B. Wang, Q. Liu, W. Xu, and T. Liao, "Exponential psynchronization of non-autonomous Cohen-Grossberg neural networks with reaction-diffusion terms via periodically intermittent control," Neural Processing Letters, vol. 40, pp. 103126, 2014.

[27] C. Li, G. Feng, and X. Liao, "Stabilization of nonlinear systems via periodically intermittent control," IEEE Transactions on Circuits and Systems II: Express Briefs, vol. 54, no. 11, pp. 10191023, 2007.

[28] S. Cai, Q. He, J. Hao, and Z. Liu, "Exponential synchronization of complex networks with nonidentical time-delayed dynamical nodes," Physics Letters A, vol. 374, no. 25, pp. 2539-2550, 2010.

[29] C. Hu, J. Yu, H. Jiang, and Z. Teng, "Exponential synchronization of complex networks with finite distributed delays coupling," IEEE Transactions on Neural Networks, vol. 22, no. 12, pp. 1999-2010, 2011.

[30] J. Mei, M. Jiang, W. Xu, and B. Wang, "Finite-time synchronization control of complex dynamical networks with time delay," Communications in Nonlinear Science and Numerical Simulation, vol. 18, no. 9, pp. 2462-2478, 2013.

[31] J. Mei, M. Jiang, B. Wang, and B. Long, "Finite-time parameter identification and adaptive synchronization between two chaotic neural networks," Journal of the Franklin Institute, vol. 350, no. 6, pp. 1617-1633, 2013.

[32] J. Mei, M. Jiang, and J. Wang, "Finite-time structure identification and synchronization of drive-response systems with uncertain parameter," Communications in Nonlinear Science and Numerical Simulation, vol. 18, no. 4, pp. 999-1015, 2013.

[33] Y. Tang, "Terminal sliding mode control for rigid robots," Automatica, vol. 34, no. 1, pp. 51-56, 1998.

[34] X. Yang and J. Cao, "Finite-time stochastic synchronization of complex networks," Applied Mathematical Modelling, vol. 34, no. 11, pp. 3631-3641, 2010.

[35] X. Liu, N. Jiang, J. Cao, S. Wang, and Z. Wang, "Finitetime stochastic stabilization for BAM neural networks with uncertainties," Journal of the Franklin Institute, vol. 350, no. 8, pp. 2109-2123, 2013.

[36] J. Mei, M. Jiang, X. Wang, J. Han, and S. Wang, "Finitetime synchronization of drive-response systems via periodically intermittent adaptive control," Journal of the Franklin Institute, vol. 351, no. 5, pp. 2691-2710, 2014. 


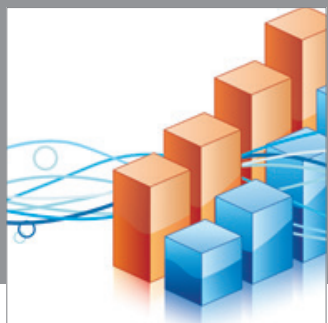

Advances in

Operations Research

mansans

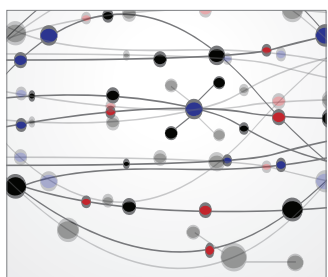

The Scientific World Journal
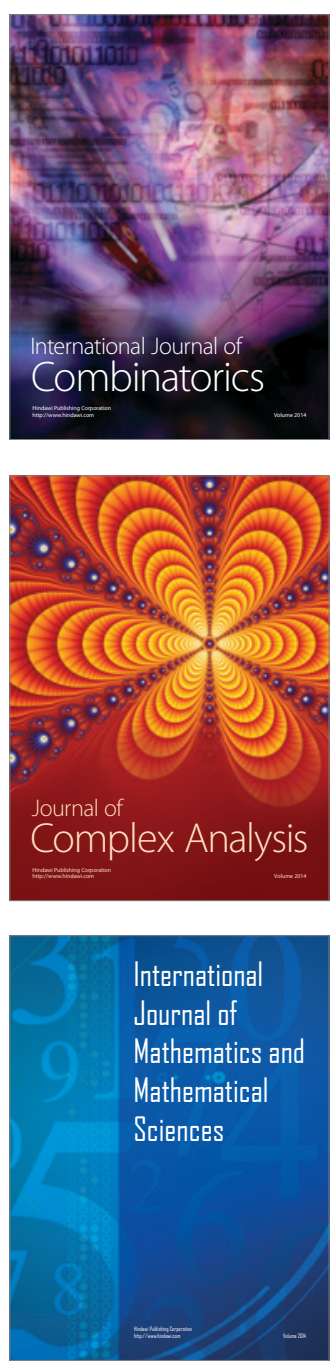


Submit your manuscripts at http://www.hindawi.com
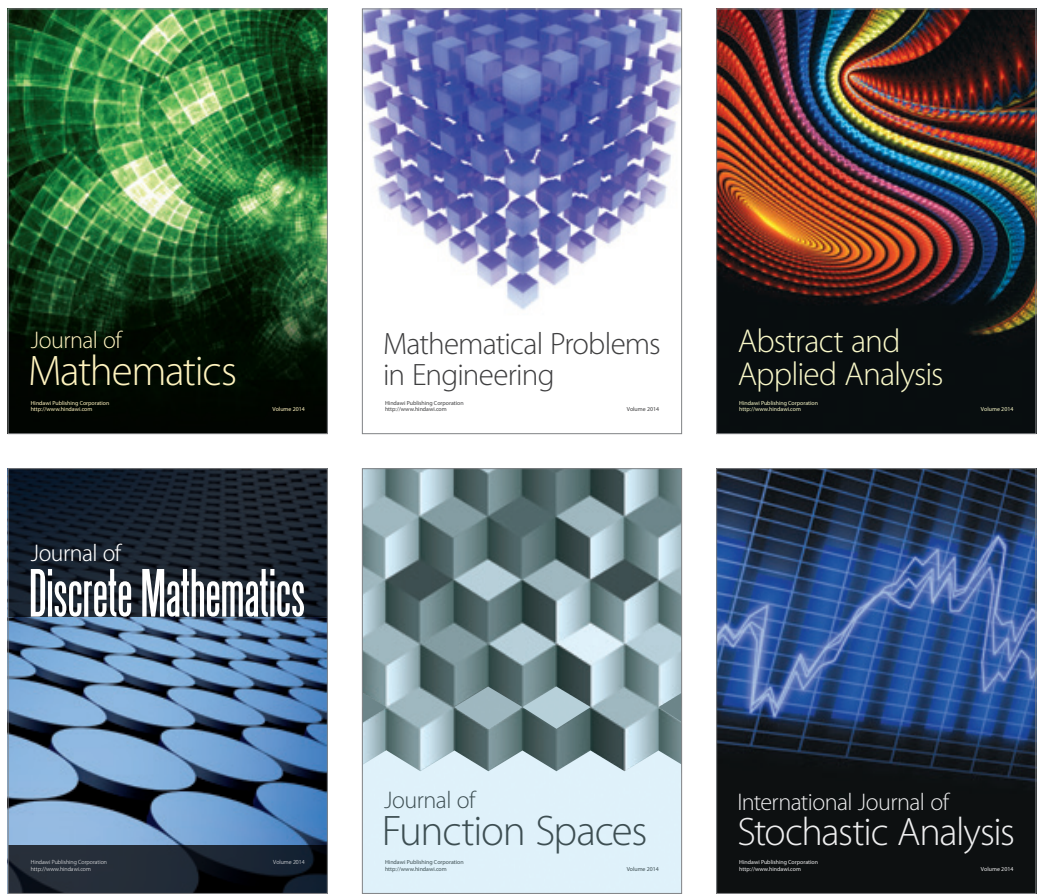

Journal of

Function Spaces

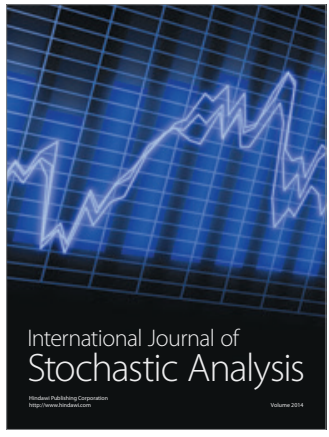

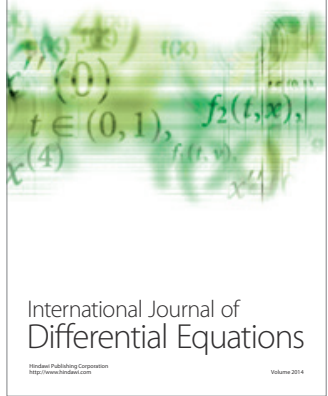
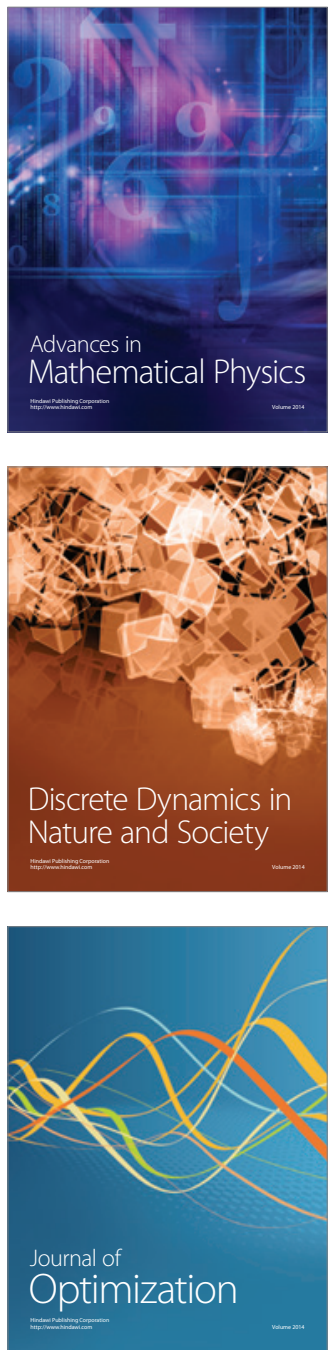